\title{
Safety and Effectiveness of Aflibercept + Fluorouracil, Leucovorin, and Irinotecan (FOLFIRI) for the Treatment of Patients with Metastatic Colorectal Cancer (mCRC) in Current Clinical Practice: OZONE Study
}

\author{
Ian Chau ${ }^{1, *(\mathbb{D}}$, Marwan Fakih ${ }^{2}$, Pilar García-Alfonso ${ }^{3}$, Zdeněk Linke ${ }^{4}$, Ana Ruiz Casado ${ }^{5}$ (D) \\ Eduardo Polo Marques ${ }^{6}$, Pascaline Picard ${ }^{7}$, Marina Celanovic ${ }^{8}$ and Thomas Cartwright ${ }^{9}$ \\ The Royal Marsden NHS Foundation Trust, London and Surrey, Sutton SM2 5PT, UK \\ City of Hope Comprehensive Cancer Center, Duarte, CA 91010, USA; mfakih@coh.org \\ Hospital General Universitario Gregorio Marañón, 28003 Madrid, Spain; pgarcaalfonso@gmail.com \\ University Hospital Motol, 15006 Prague 5, Czech Republic; Zdenek.Linke@fnmotol.cz \\ Hospital Universitario Puerta de Hierro-Majadahonda, 28222 Majadahonda, Spain; \\ anaruizcasado@gmail.com \\ 6 Miguel Servet University Hospital, 50009 Zaragoza, Spain; eduardopolomarques@hotmail.com \\ 7 Ividata, 92300 Levallois Perret, France; Pascaline.Picard-ext@sanofi.com \\ 8 Sanofi, Cambridge, MA 02139, USA; marina.celanovic@sanofi.com \\ 9 Ocala Oncology, Ocala, FL 34474, USA; tcartwright4@cox.net \\ * Correspondence: Ian.Chau@rmh.nhs.uk; Tel.: +44-208-915-6196
}

Received: 28 January 2020; Accepted: 6 March 2020; Published: 11 March 2020

\begin{abstract}
For patients with metastatic colorectal cancer (mCRC) that have failed a first-line oxaliplatin-based regimen, the preferred treatment option is an irinotecan-based regimen. This prospective, observational, noncomparative, post-authorization safety study (OZONE) evaluated the safety and effectiveness of aflibercept plus fluorouracil, leucovorin, and irinotecan (FOLFIRI) in patients with $\mathrm{mCRC}$ treated in daily practice after failure of an oxaliplatin-based regimen. Patients were grouped by age, renal impairment, hepatic impairment, race, number, and type of prior anticancer therapy. Of 766 treated patients enrolled, 59.5\% were male, $94.8 \%$ had an Eastern Cooperative Oncology Group performance status of $0-1$, all received previous chemotherapy (97.8\% including oxaliplatin), and $58.6 \%$ had prior exposure to bevacizumab. At least one grade $\geq 3$ treatment-emergent adverse event (TEAE) was reported in $68.3 \%$ of patients. Neutropenia, hypertension, diarrhea, and asthenia were the most frequently occurring grade $\geq 3$ TEAEs. Antivascular endothelial growth factor class events were infrequent. Subgroup analyses did not reveal major differences in the safety profile according to age, renal and hepatic status, race, or prior anticancer therapy. For the total population, median overall survival was 12.5 months, median progression-free survival was 6.1 months, and overall response rate was $16.3 \%$. Aflibercept in combination with FOLFIRI is a safe and efficacious regimen administered in current clinical practice to patients with mCRC previously treated with oxaliplatin. The study results, conducted in real-world clinical practice with a less selected patient population, are aligned with the VELOUR (NCT00561470) trial and no new safety issues were identified.
\end{abstract}

Keywords: aflibercept; antiangiogenic; clinical practice; FOLFIRI; metastatic colorectal cancer; observational 


\section{Introduction}

Colorectal cancer (CRC) is the third most frequently diagnosed cancer and the second leading cause of cancer-related deaths worldwide [1]. Patients with metastatic disease (mCRC) have poor outcomes with a five-year survival rate of $13.8 \%$ [2]. First-line treatment recommendations include cytotoxic combinations of fluorouracil and leucovorin with irinotecan (FOLFIRI), oxaliplatin (FOLFOX), or oxaliplatin and irinotecan (FOLFOXIRI), or capecitabine plus oxaliplatin (XELOX). Chemotherapy regimens combined with antiepidermal growth factor receptor monoclonal antibodies, such as cetuximab and panitumumab, and anti-vascular endothelial growth factor (anti-VEGF) monoclonal antibodies, such as bevacizumab, are a standard of care in the first-line treatment of mCRC [3,4]. For patients with $\mathrm{mCRC}$ who progressed on an oxaliplatin-based regimen, the preferred treatment option is an irinotecan-based regimen, usually FOLFIRI. Combining antiangiogenic agents, which target the VEGF pathway, and cytotoxic chemotherapy is a recognized treatment strategy in this setting. Clinical efficacy of FOLFIRI plus anti-VEGF agents, such as aflibercept, bevacizumab, and ramucirumab, has been demonstrated in patients with $\mathrm{mCRC}$ [5-8].

VELOUR (NCT00561470) was a Phase III randomized, placebo-controlled study investigating aflibercept plus FOLFIRI for patients with $\mathrm{MCRC}$ who had disease progression on or after an oxaliplatin-based regimen. Aflibercept plus FOLFIRI significantly improved overall survival (OS; hazard ratio $[H R]=0.817 ; p=0.0032$ ), progression-free survival (PFS; HR $=0.758 ; p<0.0001$ ), and response rate (RR; $p=0.0001$ ) versus placebo plus FOLFIRI. Based on these results, aflibercept, in combination with FOLFIRI, was approved for the treatment of patients with mCRC that are resistant to, or have progressed on, an oxaliplatin-containing regimen $[9,10]$.

Whilst randomized controlled trials (RCTs) are crucial for evaluating the efficacy and safety of medical interventions [11,12], strict enrollment criteria often lead to the exclusion of elderly patients or those with comorbidities. Consequently, RCT populations may not fully represent cancer patients receiving treatment in routine clinical practice $[12,13]$. Observational studies evaluate the safety and effectiveness of therapies in a less selected population, ultimately providing information reflecting routine clinical practice [14].

The objective of this observational post-authorization safety study (PASS), OZONE, was to assess the safety and effectiveness of aflibercept plus FOLFIRI in patients with mCRC treated in daily practice after failure of an oxaliplatin-based regimen. Subpopulations defined by age, hepatic or renal impairment, race, and number and type of prior anticancer therapy were assessed.

\section{Methods}

\subsection{OZONE Study}

OZONE was a prospective, multicenter, observational, noncomparative study evaluating patients with $\mathrm{mCRC}$ receiving aflibercept plus FOLFIRI (intravenous infusions) in the clinical setting after failure of an oxaliplatin-based regimen. Patients were followed for $\leq 24$ months from aflibercept initiation or until death. OZONE was a PASS and thus registered in the European Union electronic Register of Post-Authorization Studies (EU PAS Register ENCEPP/SDPP/4836).

\subsection{Site and Patient Selection}

Physicians prescribing aflibercept plus FOLFIRI after failure of an oxaliplatin-based regimen were randomly selected based on physicians' lists in each participating country. Independently in each country, sites offering participation in the study were randomly selected to ensure representativeness of the sample. The random process was stratified on country-specific criteria to accurately reflect routine clinical practices within each country. Patients enrolled in the study were prescribed aflibercept by their physician independently from study entry. Patients were excluded if they were participating in a clinical trial, receiving concomitant anti-VEGF agents and/or receiving aflibercept through an investigational clinical study or compassionate use program, or receiving aflibercept plus chemotherapy 
regimens other than FOLFIRI. This study was conducted in accordance with the principles outlined in the Declaration of Helsinki (18th World Medical Assembly, 1964) and all its subsequent amendments. Each patient provided signed, written, informed consent before enrolment.

\subsection{Endpoints}

There was no fixed study visit schedule, with visits occurring according to the treating physician's clinical judgment. The primary objectives were to describe safety and clinical outcomes of aflibercept plus FOLFIRI in patients treated in daily practice for mCRC after failure of an oxaliplatin-based regimen. Data were analyzed based on the overall population and on patient subgroups as follows: age ( $<65$ and $\geq 65$ years), renal function (impaired: creatinine clearance $[\mathrm{CrCl}] \leq 80 \mathrm{~mL} / \mathrm{min}$ and normal: $\mathrm{CrCl}>80 \mathrm{~mL} / \mathrm{min}$ ), hepatic function (impaired: total bilirubin $>$ upper normal limit [UNL], or aspartate transaminase [AST] or alanine aminotransferase [ALT] $>1.5$ UNL and normal: total bilirubin $\leq \mathrm{UNL}$, or AST or ALT $\leq 1.5 \mathrm{UNL}$ ), race (Caucasian and non-Caucasian), number and type of prior anticancer therapy. Secondary objectives were to describe effectiveness (e.g., PFS, OS, RR) in subgroups and to describe utilization of health resources. PFS was defined as the time from the date of the first administration of aflibercept or FOLFIRI to the date of tumor progression or death due to any cause, whichever came first. OS was defined as the time interval from the date of first administration of aflibercept or FOLFIRI to the date of death due to any cause. Best overall response during treatment period was defined as complete response (CR), partial response (PR), stable disease (SD), progressive disease (PD), or not evaluable (NE). All evaluations were performed according to the participating institution's practice and outcomes were based on the treating physician's judgment. Results of laboratory tests that were systematically performed prior to chemotherapy were not collected. The study protocol did not include specific recommendations for standardized imaging evaluation, which was performed according to investigational sites protocols.

Three categories of adverse events (AEs) were defined:

1. Pretreatment AEs: any AE reported during the pretreatment period.

2. Treatment-emergent AEs (TEAEs): an AE beginning, worsening, or becoming serious during the on-treatment period.

3. Post-treatment AEs: AEs reported during the post-treatment period.

Laboratory data collected at inclusion included a full blood count, biochemistry (including hepatic and renal function assessment), and urinary analyses. From the time period spanning from the informed consent signature to the first administration of aflibercept (pretreatment period), all AEs were collected and graded as per NCI-CTCAE version 4.03. During the interim period (post-initiation of aflibercept treatment), data was collected at approximately three-monthly ( \pm 15 days) intervals throughout the observation period for a total period of 24 months or death, whichever came first. Dose adjustments were made in accordance with the relevant prescribing information.

\section{Results}

\subsection{Study Population}

OZONE included 766 patients from 12 countries across Europe (84.3\%) and North America (15.7\%) (Table 1). Median age was 64 years, with $48.3 \%$ of patients aged $\geq 65$ years and $13.6 \%$ aged $\geq 75$ years. Most patients had an Eastern Cooperative Oncology Group performance status of 0-1 (94.8\%), and renal and hepatic impairment was present in $35.0 \%$ and $19.6 \%$ of patients, respectively. Primary tumor site was the colon for $73.5 \%$ of patients and the rectum for $25.6 \%$. All patients had metastatic disease at baseline, with $55.5 \%$ presenting with $>1$ metastatic site. Mutated KRAS and BRAF, which are genes associated with poor prognosis in CRC, were present in $51.5 \%$ and $2.7 \%$ of patients, respectively. Over a third $(35 \%)$ of patients received $>1$ prior therapy for advanced disease. All patients received previous chemotherapy ( $97.8 \%$ including oxaliplatin) and $58.6 \%$ had prior exposure to bevacizumab. Median 
time from end of treatment to inclusion was 1.0 and 4.4 months in patients treated with advanced chemotherapy and neoadjuvant/adjuvant therapy, respectively.

Table 1. OZONE patient demographics.

\begin{tabular}{|c|c|}
\hline Characteristic & Aflibercept/FOLFIRI (N = 766) \\
\hline Median age, years (range) & $64(26-88)$ \\
\hline Age $\geq 65, \%$ & 48.3 \\
\hline \multicolumn{2}{|l|}{ Gender, \% } \\
\hline Male & 59.5 \\
\hline Female & 40.5 \\
\hline ECOG PS, ${ }^{a} \%$ & $\mathrm{~N}=746$ \\
\hline 0 & 49.6 \\
\hline 1 & 45.2 \\
\hline 2 & 4.8 \\
\hline 3 & 0.4 \\
\hline Race, $\%$ & $\mathrm{~N}=762$ \\
\hline Caucasian/white & 90.8 \\
\hline Black & 4.3 \\
\hline Asian/oriental & 2.4 \\
\hline Other & 2.5 \\
\hline \multicolumn{2}{|l|}{ Country of origin, $n$} \\
\hline Czech Republic & 80 \\
\hline France & 165 \\
\hline Germany & 22 \\
\hline Greece & 34 \\
\hline Italy & 69 \\
\hline Puerto Rico & 4 \\
\hline Slovakia & 6 \\
\hline Spain & 210 \\
\hline Sweden & 14 \\
\hline Switzerland & 5 \\
\hline United Kingdom & 41 \\
\hline United States & 116 \\
\hline Renal impairment, & $\mathrm{N}=738$ \\
\hline Yes & 34.7 \\
\hline No & 65.3 \\
\hline Hepatic impairment, ${ }^{\mathrm{c}} \%$ & $N=657$ \\
\hline Yes & 19.6 \\
\hline No & 80.4 \\
\hline \multicolumn{2}{|l|}{ Prior anticancer therapy, $\%$} \\
\hline $0-1$ line & 44.8 \\
\hline$>1$ line & 55.2 \\
\hline \multicolumn{2}{|c|}{ Prior advanced disease prior anticancer therapy, $\%$} \\
\hline $0-1$ line & 65.0 \\
\hline$>1$ line & 35.0 \\
\hline \multicolumn{2}{|c|}{ Prior neoadjuvant/adjuvant chemotherapy, \% } \\
\hline Yes & 45.7 \\
\hline No & 54.3 \\
\hline \multicolumn{2}{|l|}{ Prior bevacizumab, $\%$} \\
\hline Yes & 58.6 \\
\hline No & 41.4 \\
\hline \multicolumn{2}{|l|}{ Location of primary tumor, $\%$} \\
\hline Colon & 73.5 \\
\hline Rectum & 25.6 \\
\hline Other & 0.9 \\
\hline
\end{tabular}


Table 1. Cont.

\begin{tabular}{cc}
\hline Characteristic & Aflibercept/FOLFIRI (N = 766) \\
\hline Metastases site(s), \% & \\
Liver & 67.5 \\
Lung & 51.4 \\
Lymph nodes & 22.5 \\
Peritoneum & 21.3 \\
Other & 21.0 \\
\hline KRAS status, \% & $\mathrm{N}=765$ \\
Wild type & 35.3 \\
Mutated & 51.5 \\
Unknown & 3.7 \\
Not done & 9.5 \\
\hline BRAF status, \% & \\
Wild type & 22.5 \\
Mutated & 2.7 \\
Unknown & 16.7 \\
Not done & 58.1 \\
\hline
\end{tabular}

ALT, alanine aminotransferase; AST, aspartate transaminase; $\mathrm{CrCl}$, creatinine clearance; ECOG PS, Eastern Cooperative Oncology Group performance status; FOLFIRI, fluorouracil, leucovorin, and irinotecan; UNL, upper normal limit. ${ }^{\text {a }}$ Scale used to assess patients' level of functioning in terms of their ability to care for themselves, daily activity, and physical ability; ${ }^{\mathrm{b}}$ Defined as $\mathrm{CrCl} \leq 80 \mathrm{~mL} / \mathrm{min}^{\circ}{ }^{\mathrm{c}}$ Defined as either total bilirubin > UNL or AST or ALT > 1.5 UNL.

\subsection{Treatment Exposure}

The median duration of treatment was 16.4 (range 9.6-30.0) weeks (Table 2). Patients received a median of seven cycles (range 1-46) of treatment, including a median of six cycles with aflibercept. Relative dose intensities for aflibercept, irinotecan, and fluorouracil (5-FU) were $79.0 \%, 81.4 \%$, and $82.9 \%$, respectively. A total of $277(36.2 \%)$ patients had $\geq 1$ dose reduction or delay of aflibercept.

Table 2. OZONE study treatment exposure.

\begin{tabular}{cc}
\hline Treatment exposure parameter & Aflibercept/FOLFIRI $(\mathbf{N}=\mathbf{7 6 6})$ \\
\hline Median duration of treatment, weeks (range) & $16.4(2-108)$ \\
Median number of treatment cycles, $\mathbf{n}$ (range) & $7(1-46)$ \\
Aflibercept & $6(1-44)$ \\
Irinotecan & $6(0-46)$ \\
5-FU & $6(0-46)$ \\
Median relative dose intensity, \% & 79.0 \\
Aflibercept & 81.4 \\
Irinotecan & 82.9 \\
5-FU & \\
At least one dose modification, \% & 36.2 \\
Aflibercept & 51.3 \\
Irinotecan & 67.0 \\
5-FU &
\end{tabular}

5-FU, fluorouracil; FOLFIRI, fluorouracil, leucovorin, and irinotecan.

\subsection{Safety: Overall Population}

At least one TEAE of any grade was reported in $98.3 \%$ of patients, with $68.3 \%$ of patients experiencing $\geq 1$ grade $\geq 3$ TEAE (Tables 3 and 4). Neutropenia, hypertension, diarrhea, and asthenia were the most frequently occurring grade $\geq 3$ TEAEs. Serious TEAEs were reported in $43.6 \%$ of patients, the most frequent of which were diarrhea, disease progression, and general physical health deterioration. TEAEs leading to death, excluding those related to disease progression, were reported in $14(1.8 \%)$ patients, the most frequent of which were infections $(n=5)$, nervous system disorders $(n=3)$, and gastrointestinal (GI) disorders $(n=3)$. 
Table 3. OZONE overview of treatment-emergent adverse events.

\begin{tabular}{cc}
\hline TEAE, $\%$ & Aflibercept/FOLFIRI $(\mathbf{N}=\mathbf{7 6 6})$ \\
\hline Any TEAE & 98.3 \\
Any possible related TEAE & 90.3 \\
Any grade $\geq 3$ TEAE & 68.3 \\
Any grade 5 TEAE & 7.7 \\
Any grade 3-4-related TEAE & 50.1 \\
Any serious TEAE & 43.6 \\
Any serious related TEAE & 24.2 \\
Any TEAE leading to death & 7.8 \\
\hline
\end{tabular}

FOLFIRI, fluorouracil, leucovorin, and irinotecan; TEAE, treatment-emergent adverse event.

Table 4. Summary of treatment-emergent adverse events: overall population.

\begin{tabular}{|c|c|c|}
\hline \multirow{2}{*}{ TEAE } & \multicolumn{2}{|c|}{ Aflibercept/FOLFIRI $(N=766)$} \\
\hline & All grade, $\%$ & Grade $\geq 3, \%$ \\
\hline Overall TEAEs $^{a}$ & 98.3 & 68.3 \\
\hline Diarrhea & 56.3 & 9.5 \\
\hline Asthenia & 39.6 & 9.1 \\
\hline Stomatitis & 37.9 & 5.2 \\
\hline Nausea & 33.4 & 1.3 \\
\hline Hypertension & 28.5 & 10.2 \\
\hline Neutropenia & 24.7 & 15.1 \\
\hline Decreased appetite & 22.7 & 2.7 \\
\hline Abdominal pain & 21.7 & 3.8 \\
\hline Vomiting & 21.5 & 1.8 \\
\hline Fatigue & 21.0 & 3.5 \\
\hline Epistaxis & 18.7 & 0.1 \\
\hline Constipation & 16.6 & 0.1 \\
\hline Dysphonia & 16.1 & 0.3 \\
\hline Alopecia & 12.1 & 0.4 \\
\hline Proteinuria & 11.7 & 2.7 \\
\hline Weight decreased & 11.7 & 0.3 \\
\hline Anemia & 10.7 & 1.3 \\
\hline Headache & 10.4 & 0.3 \\
\hline Overall serious TEAEs ${ }^{b}$ & 43.6 & 36.8 \\
\hline Diarrhea & 4.6 & 3.7 \\
\hline Disease progression & 3.7 & 3.7 \\
\hline $\begin{array}{c}\text { General physical health } \\
\text { deterioration }\end{array}$ & 3.3 & 3.1 \\
\hline Abdominal pain & 2.9 & 2.2 \\
\hline Intestinal obstruction & 2.5 & 2.2 \\
\hline Febrile neutropenia & 2.2 & 2.2 \\
\hline Pyrexia & 2.2 & 1.0 \\
\hline
\end{tabular}

FOLFIRI, fluorouracil, leucovorin, and irinotecan; TEAE, treatment-emergent adverse event. ${ }^{\text {a }}$ All-grade overall TEAEs reported in $\geq 10 \%$ of patients and associated grade $\geq 3$ TEAEs; ${ }^{b}$ All-grade serious TEAEs reported in $\geq 10 \%$ of patients and associated grade $\geq 3$ serious TEAEs. 
Anti-VEGF class events were infrequent, with GI perforation reported in $0.9 \%$ of patients (grouped term), GI fistula in 1.2\%, fistula from other origin in $0.8 \%$, and jaw osteonecrosis and posterior reversible encephalopathy syndrome in $0.4 \%$ each. Nephrotic syndrome was reported in one patient and no cases of thrombotic microangiopathy occurred.

\subsection{Safety: Subpopulations}

\subsubsection{Elderly}

Among the overall population, $48.3 \%(n=370)$ of patients were aged $\geq 65$ years (Table 1$)$. The rates of grade $\geq 3$ TEAEs were similar between patients aged $\geq 65$ and $<65$ years $(69.5 \%$ and $67.2 \%$, respectively) (Table $\mathrm{S} 1)$. Asthenia was more frequently reported $(\geq 5 \%)$ in elderly versus younger patients.

\subsubsection{Impaired Renal Function}

Among patients with renal status documented at baseline $(N=738), 34.9 \%(n=258)$ had impaired renal function $(\mathrm{CrCl} \leq 80 \mathrm{~mL} / \mathrm{min})$ (Table 1$)$. Of patients defined as renally impaired, $16.7 \%(n=43)$ and $83.3 \%(n=215)$ had $\mathrm{CrCl}<50 \mathrm{~mL} / \mathrm{min}$ and $50-80 \mathrm{~mL} / \mathrm{min}$, respectively. Rates of all-grade and grade $\geq 3$ TEAEs were similar between patients with and without renal impairment (Table S2).

In patients with $\mathrm{CrCl}<50 \mathrm{~mL} / \mathrm{min}$, grade $\geq 3$ proteinuria was reported more frequently compared with the other two subgroups, whilst grade $\geq 3$ asthenia was reported more frequently versus patients with $\mathrm{CrCl}>80 \mathrm{~mL} / \mathrm{min}$. Hypertension was reported more frequently in the $\mathrm{CrCl} 50-80 \mathrm{~mL} / \mathrm{min}$ and $\mathrm{CrCl}>80 \mathrm{~mL} / \mathrm{min}$ groups compared with the $\mathrm{CrCl}<50 \mathrm{~mL} / \mathrm{min}$ group (Table S2).

\subsubsection{Impaired Liver Function}

Among patients with liver status documented at baseline $(N=657), 19.6 \%(n=129)$ had impaired liver function (total bilirubin $>$ UNL, or $>1.5 \mathrm{UNL}$ ) (Table 1). No grade $\geq 3$ TEAEs were reported more frequently ( $\geq 5 \%$ ) in either group (Table S3).

\subsubsection{Non-Caucasian}

Among patients with race documented at baseline $(N=762), 9.2 \%(n=70)$ were non-Caucasian (Table 1). No grade $\geq 3$ TEAEs were reported more frequently ( $\geq 5 \%$ ) in either group (Table S4).

\subsubsection{Received $>1$ Prior Anticancer Therapy}

Among the overall population, 55.2\% $(n=423)$ of patients had received $>1$ prior anticancer therapy (Table 1). Grade $\geq 3$ asthenia was reported more frequently in the $>1$ prior line subgroup (Table S5).

\subsubsection{Prior Exposure to Bevacizumab}

Among the overall population, 58.6\% $(n=449)$ of patients had received prior treatment with bevacizumab (Table 1 ). Only grade $\geq 3$ hypertension was more frequently reported in patients who had received no prior bevacizumab (Table S6).

\subsection{Efficacy}

For the overall population, median OS was 12.5 months (Figure 1A), median PFS was 6.1 months (Figure S1), and overall RR (ORR) was 16.3\% (Table 5). A CR was reported in 10 (1.3\%) patients and $115(15.0 \%)$ patients reported a PR (Figure 2). Multivariate analyses showed no clinically meaningful differences between groups defined by ages, race, baseline renal function, or number of prior lines of chemotherapy. Patients with hepatic impairment had shorter OS (8.7 vs. 13.7 months; HR [95\% confidence interval $\{\mathrm{CI}\}]$ : 1.56 [1.26-1.94]), PFS (4.4 vs. 6.3 months; HR [95\% CI]: 1.42 [1.16-1.75]), 
and ORR (10.9\% vs. $17.0 \%$; odds ratio [OR; $95 \%$ CI]: 0.63 [0.33-1.17]) versus those without hepatic impairment (Figure 1D, Figure S1D). Median OS in patients with prior bevacizumab therapy was 10.6 months versus 16.6 months in those with no prior bevacizumab treatment (HR [95\% CI]: 1.67 [1.38-2.01]). Respective PFS and ORR were 5.2 versus 7. 5 months (HR [95\% CI]: 1.59 [1.33-1.88]) and $11.5 \%$ versus $20.6 \%$ (OR [95\% CI]: 0.51 [0.34-0.78]) (Figure 1G, Figure S1G). Patients aged < 65 years had lower ORR versus those $\geq 65$ years (11.5\% vs $19.2 \%$; OR [95\% CI]: 1.84 [1.21-2.81]) (Table 5). Similarly, patients without renal impairment had a numerically lower ORR versus those with renal impairment (13.5\% vs. $19.4 \%$; OR [95\% CI]: 1.52 [0.99-2.33]).

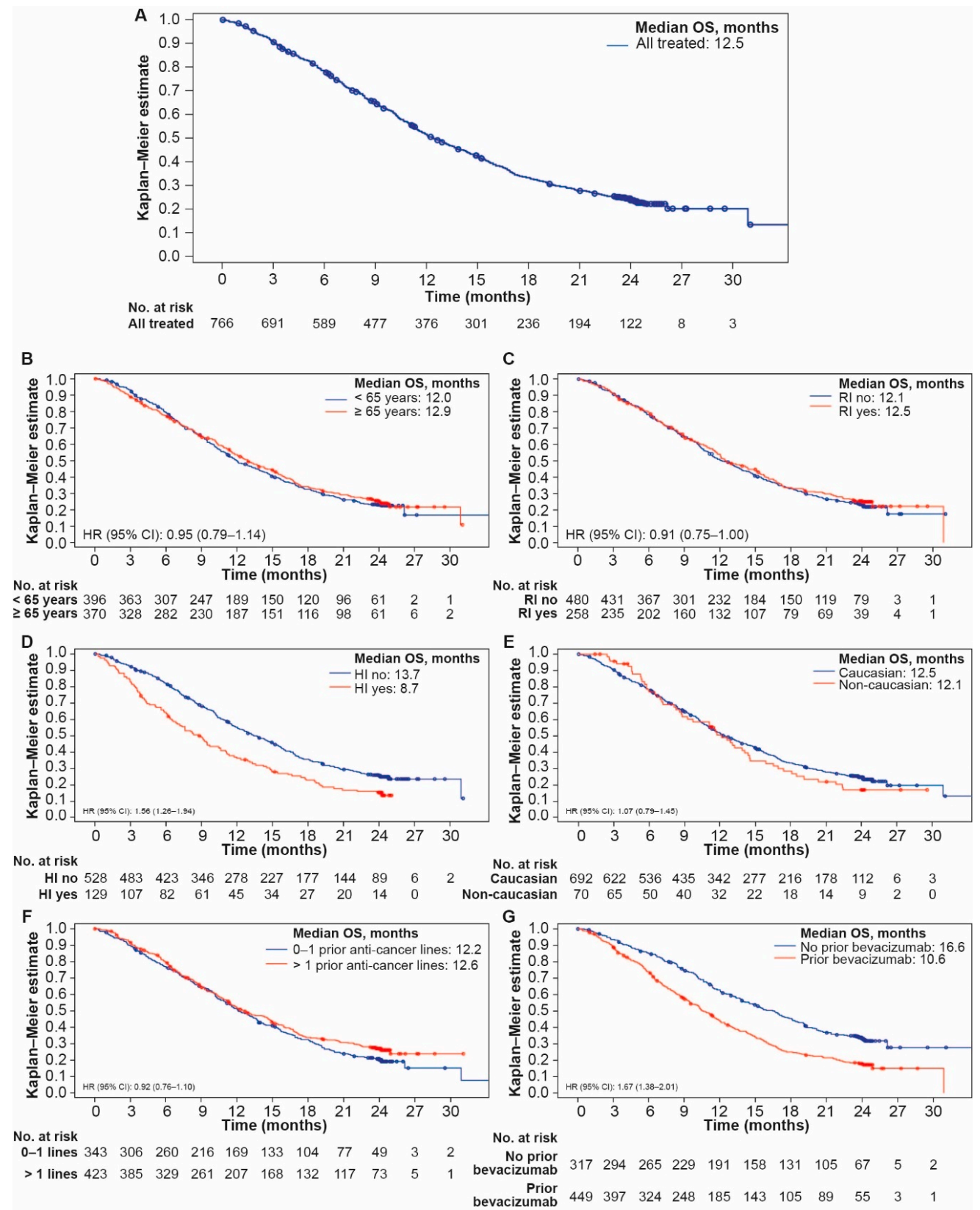

Figure 1. Overall survival (A) for the overall treated population; (B) according to age ( $<65 / \geq 65$ years); (C) according to renal impairment (yes/no); (D) according to hepatic impairment (yes/no); (E) according to race (Caucasian/non-Caucasian); (F) according to prior anticancer therapy (0-1/> 1 lines); (G) according to prior use of bevacizumab (yes/no).CI, confidence interval; HI, hepatic impairment; HR, hazard ratio; OS, overall survival; RI, renal impairment. 
Table 5. Overall response rate multivariate analyses for subgroups of interest.

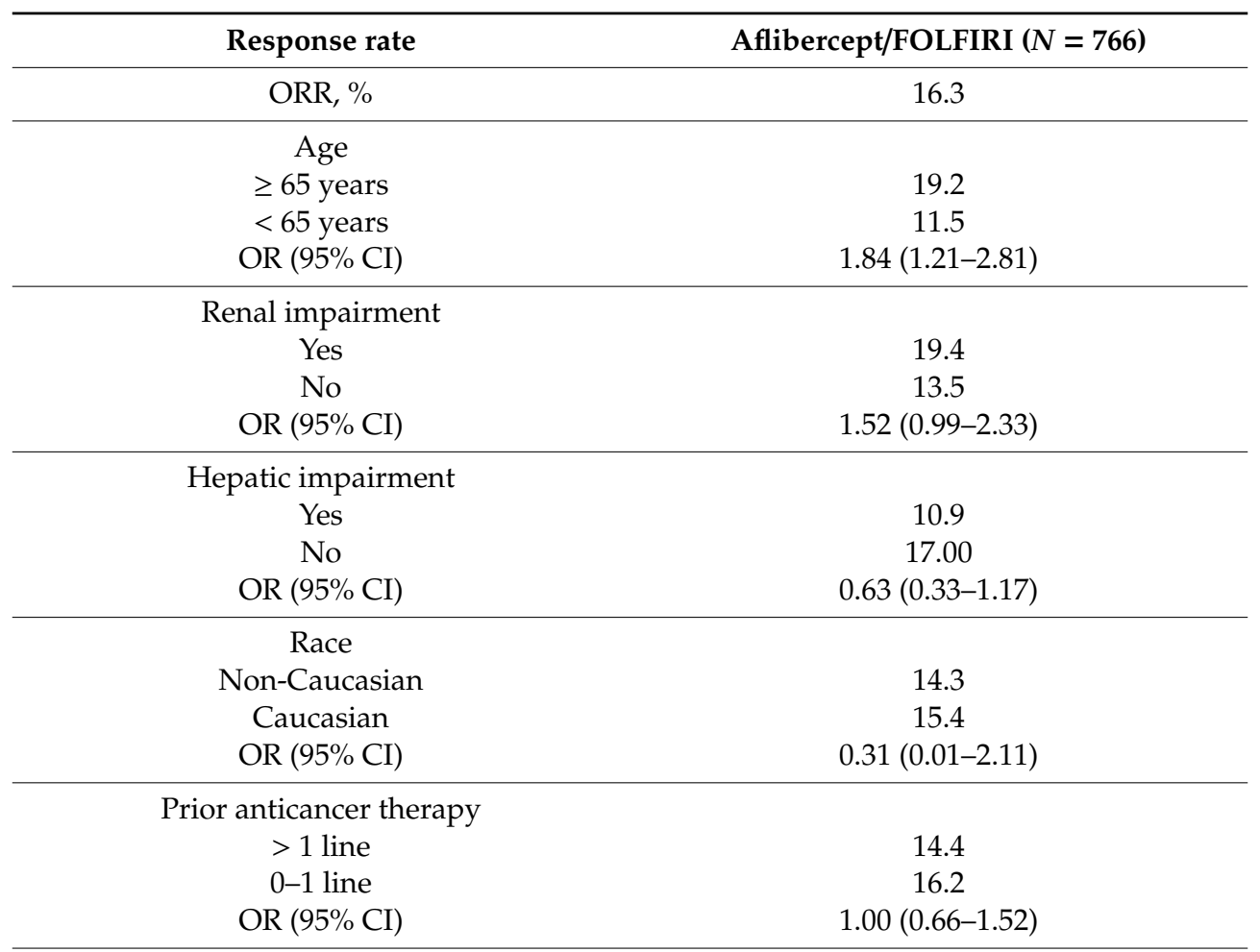

Prior bevacizumab therapy

Yes $\quad 11.5$

No

20.6

OR $(95 \% \mathrm{CI})$

$0.51(0.34-0.78)$

$\mathrm{CI}$, confidence interval; FOLFIRI, fluorouracil, leucovorin, and irinotecan; HR, hazard ratio; OR, odds ratio; ORR, overall response rate.

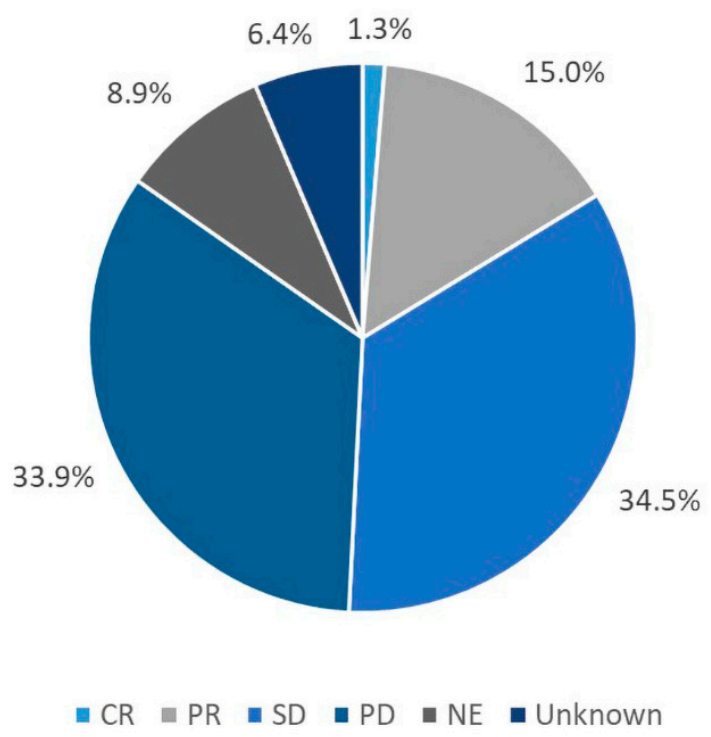

Figure 2. OZONE summary of overall response rate.CR, complete response; NE, not evaluable; PD, progressive disease; $\mathrm{PR}$, partial response; $\mathrm{SD}$, stable disease.

\section{Discussions}

In the VELOUR trial, aflibercept plus FOLFIRI was associated with prolonged OS and PFS versus placebo plus FOLFIRI in patients with mCRC [5]. Whilst studies such as these are required for 
registration purposes, Phase III RCTs have limitations, particularly when considering the rigorously controlled patient populations that are recruited, which often do not closely reflect patients in the clinic. PASSs evaluate the safety and effectiveness of therapies in a comparatively less selected population and are therefore useful for extending the knowledge of recently approved compounds in real-life practice, providing complementary information to formal clinical development [11,12,15-19]. OZONE evaluated the use of aflibercept plus FOLFIRI in routine clinical practice in patients with mCRC after failure of an oxaliplatin-based regimen, mirroring real-life patient management. The trial confirms that aflibercept in combination with FOLFIRI is a safe and efficacious regimen administered in current clinical practice to patients with mCRC previously treated with oxaliplatin. The study results, conducted in real-world clinical practice with a less selected patient population, are aligned with the VELOUR (NCT00561470) trial and no new safety issues were identified.

Patients in the OZONE study were older than those in the VELOUR study (median 64 years, $48.3 \%$ aged $\geq 65$ years versus median 61 years, $33.5 \%$ aged $\geq 65$ years), although other disease characteristics did not differ. The proportion of patients who had neoadjuvant/adjuvant chemotherapy and who had prior exposure to bevacizumab was higher in OZONE versus VELOUR $(45.7 \%$ vs. $26.5 \%$ and $58.6 \%$ vs. $27.6 \%$, respectively), which is reflective of the difference in time between the initiation of both trials. Furthermore, patients in VELOUR had received only one prior line of therapy for metastatic disease. The OZONE population could have received $>1$ line of therapy $(55.2 \%$ had received $>1$ prior line) and thus was consistent with the patients enrolled on more recent, real-world studies [15-19].

No new safety concerns emerged from this study. Diarrhea, asthenia, stomatitis, nausea, and hypertension were the most frequently occurring TEAEs of any grade, consistent with VELOUR and previously published real-world studies [5,15-19]. Anti-VEGF class effects were uncommon, and rates remained similar to those reported in VELOUR.

Real-world studies also provide an opportunity to evaluate specific patient populations. Subgroup analysis of the OZONE study did not show major differences in the safety profile according to age, renal and hepatic status, race, or prior anticancer therapy. This supports the use of aflibercept in patients who are older and have renal or hepatic impairment, and independent of race or previous treatment with bevacizumab. However, these results should be interpreted with caution due to the small patient numbers investigated in several of the subgroups $(n=43$ with $\mathrm{CrCl}<50 \mathrm{~mL} / \mathrm{min} ; n=70$ non-Caucasian; $n=129$ with hepatic impairment).

OZONE results support the conclusions of VELOUR and real-world studies, that aflibercept is a valuable treatment option for patients with mCRC [5,15-19]. When focusing on patient subgroups, OS multivariate analyses showed that treatment with aflibercept favored the subgroups of patients with no hepatic impairment and no prior use of bevacizumab. The observed numerical difference in median OS based on hepatic function may be impacted by the larger proportion of patients with baseline liver metastasis in the hepatic impairment subgroup (79.8\% vs. $64.8 \%$ in patients with vs. without impaired hepatic function) and a greater proportion with metastatic disease at diagnosis (81.4\% vs. $66.7 \%)$. Outcomes in patients who had prior bevacizumab (median OS: 10.6 months) are consistent with that reported in VELOUR (12.5 months) [20]. In real-world studies, the impact of prior bevacizumab is not as clear. In one study, prior bevacizumab was associated with numerically shorter OS and PFS [15]. However, the Spanish Named Patient Programme reported that prior bevacizumab was associated with improved PFS [17]. Restriction of antiepidermal growth factor receptor treatment to the wild-type KRAS population was implemented after the last patient completed treatment in the VELOUR study. This landmark change in the treatment of patients with mCRC may have contributed to the favorable 16.62-month median OS in the group of patients with no prior bevacizumab by selecting a wild-type KRAS population within this subgroup.

OZONE results further support the value of aflibercept for patients with $\mathrm{mCRC}$ in routine clinical practice and are consistent with what has previously been reported in a formal registration Phase III study [5]. However, there are some limitations, which are common to this type of study and must be considered. Although centers were selected at random, there are limitations regarding 
representativeness of the selected sites. No standardized method for evaluating efficacy could be used in the OZONE study, which affects the interpretation of RR and PFS. This should be considered when comparing with results from the VELOUR study, in which imaging evaluation was performed more frequently than in real-life practice. Results of the laboratory tests that were systematically performed prior to administration of chemotherapy were not collected in OZONE electronic case report forms, precluding comparison of hematologic toxicity with VELOUR.

\section{Conclusions}

In conclusion, OZONE confirms that aflibercept in combination with FOLFIRI is a safe and efficacious regimen administered in current clinical practice to patients with $\mathrm{mCRC}$ previously treated with oxaliplatin. The study results, conducted in real-world clinical practice with a less selected patient population, are aligned with the VELOUR (NCT00561470) trial and no new safety issues were identified.

Supplementary Materials: The following are available online at http://www.mdpi.com/2072-6694/12/3/657/s1, Figure S1: Progression-free survival A) for the overall treated population; B) according to age ( $<65 / \geq 65$ years); C) according to renal impairment (yes/no); D) according to hepatic impairment (yes/no); E) according to race (Caucasian/non-Caucasian); F) according to prior anticancer therapy (0-1/>1 lines); G) according to prior use of bevacizumab (yes/no), Table S1: Summary of treatment-emergent adverse events: by age, Table S2: Summary of treatment-emergent adverse events: by renal impairment, Table S3: Summary of treatment-emergent adverse events: by hepatic impairment, Table S4: Summary of treatment-emergent adverse events: by race, Table S5: Summary of treatment-emergent adverse events: by prior anticancer therapy, Table S6: Summary of treatment-emergent adverse events: by prior bevacizumab treatment.

Author Contributions: Conceptualization, all authors; Methodology, P.P. and M.C.; Validation, P.P. and M.C.; Formal Analysis, P.P. and M.C.; Writing-Review \& Editing, all authors. All authors have read and agreed to the published version of the manuscript.

Funding: This study was funded by Sanofi U.S. LLC. The authors were responsible for all content and editorial decisions and received no honoraria related to the development of this publication. Editorial support was provided by Sophie McEntegart of MediTech Media, funded by Sanofi U.S. LLC.

Acknowledgments: Chau would like to acknowledge National Health Service funding to the National Institute for Health Research Biomedical Research Centre at the Royal Marsden NHS Foundation Trust and The Institute of Cancer Research.

Conflicts of Interest: IC has participated in advisory boards for Eli-Lilly, Bristol-Myers Squibb, MSD, Bayer, Roche, Merck Serono, Five Prime Therapeutics, Astra Zeneca, Oncologie International, and Pierre Fabre, has received research funding from Eli-Lilly, Janssen-Cilag, Sanofi Oncology, and Merck Serono, and has received honorarium from Eli-Lilly. MF has received personal fees from Amgen, Array BioPharma, and Bayer, has received grants from Amgen, Astra Zeneca, and Novartis, and has received personal fees from Seattle Genetics. PG-A has participated in advisory boards for Amgen, Bristol-Myers Squibb, MSD, Roche, Merck Serono, Sanofi Oncology, Ipsen, and Servier. ZL received personal fees from Sanofi, Amgen, and Servier, and travel support from Merck. ARC has received personal fees from Servier, Sanofi, Amgen, Merck, Eli-Lilly, Roche, Medtronic, and BTG, and grants from Sanofi. EPM has received personal fees from Roche, Amgen, Sanofi, and Merck Serono. PP is employed by Ividata on behalf of Sanofi. MC is employed by Sanofi. TC has received personal fees from Amgen and Taiho.

\section{References}

1. Bray, F.; Ferlay, J.; Soerjomataram, I.; Siegel, R.L.; Torre, L.A.; Jemal, A. Global cancer statistics 2018: GLOBOCAN estimates of incidence and mortality worldwide for 36 cancers in 185 countries. CA Cancer J. Clin. 2018, 68, 394-424. [CrossRef] [PubMed]

2. Noone, A.M.; Howlader, N.; Krapcho, M. SEER Cancer Statistics Review, 1975-2015; Based on November 2017 SEER data submission, posted to the SEER website April 2018; National Cancer Institute: Bethesda, MD, USA, 2018. Available online: https://seer.cancer.gov/archive/csr/1975_2015/ (accessed on 1 February 2020).

3. Fakih, M.G. Metastatic colorectal cancer: Current state and future directions. J. Clin. Oncol. 2015, 33, 1809-1824. [CrossRef] [PubMed]

4. NCCN Clinical Practice Guidelines in Oncology (NCCN Guidelines); Colon Cancer: 2019; Version 2; Harborside Press, LLC,: Cold Spring Harbor, NY, USA, 2019. 
5. Van Cutsem, E.; Tabernero, J.; Lakomy, R.; Prenen, H.; Prausová, J.; Macarulla, T. Addition of aflibercept to fluorouracil, leucovorin, and irinotecan improves survival in a phase III randomized trial in patients with metastatic colorectal cancer previously treated with an oxaliplatin-based regimen. J. Clin. Oncol. 2012, 30, 3499-3506. [CrossRef] [PubMed]

6. Bennouna, J.; Hiret, S.; Bouché, O.; Deplanque, G.; Borel, C.; François, E.; Conroy, T.; Ghiringhelli, F.; des Guetz, G.; Bennouna, J.; et al. Continuation of bevacizumab vs cetuximab plus chemotherapy after first progression in KRAS wild-type metastatic colorectal cancer: The UNICANCER PRODIGE18 randomized clinical trial. JAMA Oncol. 2019, 5, 83-90. [CrossRef] [PubMed]

7. Bennouna, J.; Hiret, S.; Bertaut, A.; Bouché, O.; Deplanque, G.; Borel, C. Continuation of bevacizumab after first progression in metastatic colorectal cancer (ML18147): A randomised phase 3 trial. Lancet Oncol. 2013, 14, 29-37. [CrossRef]

8. Tabernero, J.; Yoshino, T.; Cohn, A.L.; Obermannova, R.; Bodoky, G.; Garcia-Carbonero, R. Ramucirumab versus placebo in combination with second-line FOLFIRI in patients with metastatic colorectal carcinoma that progressed during or after first-line therapy with bevacizumab, oxaliplatin, and a fluoropyrimidine (RAISE): A randomised, double-blind, multicentre, phase 3 study. Lancet Oncol. 2015, 16, 499-508. [PubMed]

9. Zaltrap (ziv-aflibercept) US Prescribing Information; Sanofi-aventis U.S. LLC: Bridgewater, NJ, USA, 2012.

10. Sanofi. ZALTRAPC (ziv-aflibercept) Injection, Summary of Product Characteristics; EMA: Amsterdam, The Netherlands, 2017.

11. Kennedy-Martin, T.; Curtis, S.; Faries, D.; Robinson, S.; Johnston, J. A literature review on the representativeness of randomized controlled trial samples and implications for the external validity of trial results. Trials 2015, 16, 495. [CrossRef] [PubMed]

12. Carroll, R.; Ramagopalan, S.V.; Cid-Ruzafa, J.; Lambrelli, D.; McDonald, L. An analysis of characteristics of post-authorisation studies registered on the ENCePP EU PAS Register. F1000Research 2017, 6, 1447. [CrossRef] [PubMed]

13. Cohen, A.T.; Goto, S.; Schreiber, K.; Torp-Pedersen, C. Why do we need observational studies of everyday patients in the real-life setting? Eur. Heart J. 2015, 17, D2-D8. [CrossRef]

14. Booth, C.M.; Tannock, I.F. Randomised controlled trials and population-based observational research: Partners in the evolution of medical evidence. Br. J. Cancer 2014, 110, 551-555. [CrossRef] [PubMed]

15. Fernández Montes, A.; Martínez Lago, N.; Covela Rúa, M.; de la Cámara Gómez, J.; González Villaroel, P.; Méndez Méndez, J.C. Efficacy and safety of FOLFIRI/aflibercept in second-line treatment of metastatic colorectal cancer in a real-world population: Prognostic and predictive markers. Cancer Med. 2019, 8, 882-889. [CrossRef] [PubMed]

16. Ivanova, J.I.; Saverno, K.R.; Sung, J.; Duh, M.S.; Zhao, C.; Cai, S. Real-world treatment patterns and effectiveness among patients with metastatic colorectal cancer treated with ziv-aflibercept in community oncology practices in the USA. Med. Oncol. 2017, 34, 193. [CrossRef] [PubMed]

17. Feliu, J.; de Corcuera, I.D.; Manzano, J.L.; Valladares-Ayerbes, M.; Alcaide, J.; García, T.G. Effectiveness and safety of aflibercept for metastatic colorectal cancer: Retrospective review within an early access program in Spain. Clin. Transl. Oncol. 2017, 19, 498-507. [CrossRef] [PubMed]

18. Pastorino, A.; Di Bartolomeo, M.; Maiello, E.; Iaffaioli, V.; Ciuffreda, L.; Fasola, G. Aflibercept Plus FOLFIRI in the Real-life Setting: Safety and Quality of Life Data From the Italian Patient Cohort of the Aflibercept Safety and Quality-of-Life Program Study. Clin. Colorectal. Cancer 2018, 17, e457-e470. [CrossRef] [PubMed]

19. Gouverneur, A.; Claraz, P.; Rousset, M.; Arnaud, M.; Fourrier-Réglat, A.; Pariente, A. Comparative Safety of Targeted Therapies for Metastatic Colorectal Cancer between Elderly and Younger Patients: A Study Using the International Pharmacovigilance Database. Target Oncol. 2017, 12, 805-814. [CrossRef] [PubMed]

20. Tabernero, J.; Van Cutsem, E.; Lakomý, R.; Prausová, J.; Ruff, P.; Van Hazel, G.A. Aflibercept versus placebo in combination with fluorouracil, leucovorin and irinotecan in the treatment of previously treated metastatic colorectal cancer: Prespecified subgroup analyses from the VELOUR trial. Eur. J. Cancer 2014, 50, 320-331. [CrossRef] [PubMed]

(C) 2020 by the authors. Licensee MDPI, Basel, Switzerland. This article is an open access article distributed under the terms and conditions of the Creative Commons Attribution (CC BY) license (http://creativecommons.org/licenses/by/4.0/). 